\title{
La Cátedra del Adulto Mayor en la provincia de Mayabeque: materialización del trabajo extensionista
}

\section{Milagros de la C. Socas Reinoso}

Universidad Agraria de La Habana "Fructuoso Rodríguez Pérez" | Cuba sara@unah.edu.cu

\section{Alexis Torres Alonso}

Universidad Agraria de La Habana "Fructuoso Rodríguez Pérez" | Cuba alexist@unah.edu.cu

\section{Resumen}

Como parte de la labor extensionista desde la universidad hacia la comunidad surgen las Cátedras del Adulto Mayor (CUAM). En ellas las personas de la tercera edad se preparan para una vejez responsable y plena. El presente artículo tiene como objetivo presentar la experiencia de la Universidad Agraria de La Habana (UNAH), en el funcionamiento de dicha cátedra en la provincia de Mayabeque. Se han tomado como referentes los resultados de la encuesta aplicada a los coordinadores y los trabajos presentados por sus miembros en las ediciones del evento GERONTOHABANA, donde se demuestra el trabajo sociocultural universitario que ha logrado desarrollarse en el territorio con la participación de los adultos mayores como promotores del proceso de extensión.

\section{Palabras clave}

Cátedra del Adulto Mayor; Gerontología educativa; Trabajo Sociocultural Universitario. 


\section{Introducción}

La longevidad como capacidad de expansión de la vida se ha incrementado en el mundo. Según datos de la Organización Mundial de Salud (OMS, 1999) en 1950 habían 200 millones de personas de más de 60 años en el mundo; en 1975 aumentó en 350 millones. Se estima que para el 2000 aumentará a 590 millones y para el 2025 habrán 1100 millones de personas de edad avanzada en el planeta y constituirán el 13.7\% de la población mundial.

Como plantea Fariñas (2013), el envejecimiento poblacional, es un nuevo desafío que ocupa las agendas de los estados a nivel mundial, es un fenómeno palpable en las sociedades, con sus consecuencias e impacto sobre la salud, la familia, la actividad económica y los presupuestos de seguridad y asistencia social. Cuba y su Revolución no están exceptas de este fenómeno demográfico, la calidad de vida de los cubanos a partir de 1959 se elevó a causa de los principales programas revolucionarios, en particular el de salud para todos.

Datos estadísticos sitúan a Cuba como uno de los países más envejecidos de América Latina y está previsto que hacia el 2050, sea uno de los más envejecidos del mundo con 2 041392 adultos mayores en su población y al igual que países desarrollados muestra elevados indicadores de salud, educación y de seguridad social. Todo lo anterior indica que se hace necesario prestar especial atención a este sector social para poder atenderlo de acuerdo a sus particularidades y que ellos mismos puedan asumir una vejez responsable.

En este entorno la universidad también acometió acciones para cumplir con una nueva misión en su encargo social. A partir del año 2000 comienza el proceso de Universalización de la Educación Superior Cubana en los municipios, como continuidad de la revolución educacional que se gestó desde 1959. En este marco surgen las Sedes Universitarias Municipales (SUM), que posteriormente con el proceso de integración de los organismos formadores devinieron en Centros Universitarios Municipales(CUM), adscriptos a las universidades del Ministerio de Educación Superior (MES) de cada una de las provincias. Es en estos espacios y con la atención directa de la universidad que surgen las Cátedras Universitarias del Adulto Mayor (CUAM), que contribuye a la atención del adulto y la satisfacción de sus necesidades de participación social.

En la provincia Mayabeque se desarrollan 11 Filiales Universitarias del Adulto Mayor, en las mismas desde los presupuestos teóricos de la Andragogía y Gerontagogía, se imparten cursos que permiten dotar a los participantes de conocimientos sólidos para asumir con responsabilidad y participación ciudadana esta nueva etapa de la vida. A más de 12 cursos de creada, se pueden identificar logros alcanzados en el trabajo así como algunas deficiencias que deben ser atendidas en aras de perfeccionar este proceso formativo. El presente trabajo tiene 
como objetivo realizar un análisis crítico del desarrollo de las Filiales Universitarias Municipales en la provincia Mayabeque, atendidas por la Universidad Agraria de La Habana.

\section{Referentes teóricos en los que se sustenta la Cátedra Universitaria del Adulto Mayor en Cuba}

En la provincia La Habana, la Cátedra del Adulto Mayor se crea a partir del 2002, destacándose por el incremento en su matrícula cada año y la calidad de su funcionamiento, siendo de referencia a nivel nacional. En el pasado curso 2013-2014, ya dividida la provincia y constituida Mayabeque, se contaba con 52 aulas correspondientes a las 11 Filiales de la CUAM, con un total de 523 cursantes, y más de 331 profesores, quienes de manera voluntaria han ofrecido por años sus conocimientos a favor de la superación cultural de las personas mayores cubanas de su comunidad. Se ha logrado llegar con este programa a zonas urbanas y rurales en los municipios cabeceras, funcionan aulas donde estén creadas las condiciones: universidades, escuelas, casas de cultura, hogares de ancianos, centros penitenciarios, fábricas, museos, centros de salud, entre otros.

La base teórica y conceptual del funcionamiento de la CUAM, parte de asumir la adultez mayor desde el punto de vista psicológico como una etapa del desarrollo de la personalidad y del aprendizaje, para ello han de sustentarse en los referentes teóricos que desde las Ciencias de la Educación, garantizan un adecuado proceso educativo y de aprendizaje en esta edad, partiendo de algunos postulados (OROSA, 2001):

- La Psicología del envejecimiento y la vejez

- Psicología del Desarrollo desde la perspectiva Histórico Cultural.

- La Andragogía como rama de la educación de adultos.

- La Gerontagogía como aplicación de las Ciencias de la Educación a la Gerontología.

- El Lifelong Learning o Educación a lo largo de la vida.

La expansión y consolidación de la educación de adultos mayores como un campo teórico-práctico diferenciado dentro del ámbito educativo, ha generado reiteradas discusiones acerca de la posibilidad de conformar una subdisciplina de carácter científico. Algunos consideran que en tanto teoría y práctica educativa debe incluirse dentro del campo de las Ciencias de la Educación, mientras que para otros autores, su lugar de adscripción, es el campo de la Gerontología como ámbito multidisciplinario dedicado al estudio de los procesos de envejecimiento y la vejez. Esta discusión remite a diferentes formas de nominar las prácticas educativas con adultos mayores, tales como: Andragogía, Geragogía, Gerontagogía o simplemente educación de adultos mayores. 
A comienzos de los ochenta se difunde ampliamente en el campo de la educación de adultos, un enfoque pedagógico para la educación de adultos, desarrollado por Malcom Knowles (1980, 1983), que denominó Andragogía. Con este nombre quiso especificar un ámbito conceptual centrado en las características del adulto, como base para la intervención educativa. En sus palabras, la Andragogía es definida como el "arte y la ciencia de ayudar a los adultos a aprender" (KNOWLES, 1980).

Según Flecha (1994) y Jarvis (1985) hay cuatro rasgos que especifican el aprendizaje adulto tal como lo concibe la Andragogía: 1) El auto-concepto de una persona pasa de la dependencia a la auto-dirección a medida que está madura. 2) Cuando el sujeto avanza en edad acumula un fondo de experiencias que constituyen su principal recurso para el aprendizaje. 3) La disposición para aprender en la adultez se sustenta en las necesidades que le impone el cumplimiento de roles sociales. 4) El conocimiento es aprendido para ser aplicado en el corto plazo y por lo tanto el aprendizaje se debe centrar más en la resolución de problemas que en el desarrollo del sujeto.

La perspectiva Andragógica sentó las bases para un modelo de educación de adultos centrado en el aprendiente. En las etapas iniciales de la institucionalización de la educación de adultos mayores, este modelo ha tenido cierto influjo en el contexto anglosajón. Para fundamentar e implementar intervenciones educativas orientadas a las personas mayores este modelo ha revalorizado los intereses del mayor, el papel que la acumulación de la experiencia tiene en el direccionamiento de la motivación y el mantenimiento de las metas de aprendizaje y, finalmente, la perspectiva que los años le otorgan para evaluar el valor del aprendizaje nuevo.

Por ello se considera oportuno que sean comprendidos y estudiados los principales presupuestos de la Andragogía (TURNER, 2001):

- Diferenciar los modelos pedagógicos y andragógicos.

- Partir de las necesidades de qué aprender en esta edad, ¿qué se quiere aprender, cuáles son las historias personales de los cursantes, sus vivencias, y cómo quiere aprender?

- Considerar la relación horizontal participativa adulto-adulto, y no maestro-alumno, ambos constituyen lugares del saber.

- El profesor como facilitador, y el participante como corresponsable de su aprendizaje.

- Desarrollar el proceso educativo no como proceso de enseñanza- aprendizaje, sino de orientación-aprendizaje.

- Garantizar la interacción entre los participantes o estudiantes.

- Abordar el proceso evaluativo como autoevaluación o evaluación conjunta por subgrupos (o también llamados familias). 
A comienzos de los años setenta comenzaron a desarrollarse iniciativas que instituyeron nuevas prácticas educativas con personas mayores. Estas trascienden los objetivos usuales de alfabetización o post-alfabetización y adoptan una perspectiva marcadamente generacional. La aparición de modalidades educativas innovadoras como la Open University en Inglaterra, las Universidades de la Tercera Edad en Francia y las U3A en Inglaterra, y el creciente flujo de personas mayores hacia estas y otras iniciativas, produjo un entrecruzamiento entre la gerontología social y la educación que sentó las bases para la delimitación de este campo disciplinar.

Varios autores ubican en este momento la aparición de un nuevo campo de estudio y de intervenciones pedagógicas: la Gerontología Educativa (Martín García, 1995; Glendenning, 1990). Una definición de la Gerontología Educativa considerada clásica postula que ésta "se refiere al estudio y práctica de emprendimientos educativos para y acerca de las personas de edad y del envejecimiento" (PETERSON, 1976). Como puede observarse en esta definición, el autor establece una relación dialéctica entre los campos de la práctica y teorización referidos tanto a las acciones educativas orientadas a los adultos mayores, como a otros públicos interesados en el conocimiento de la vejez y el envejecimiento en sus múltiples derivaciones.

En la década del ochenta Peterson (1980) acuñó el término Gerontología Educativa para referirse a un subcampo de la Gerontología centrado en las relaciones entre la educación y el proceso de envejecimiento. Actualmente se entiende la Gerontología Educativa como el estudio y la práctica de emprendimientos educativos dirigidos a las personas mayores (educación en la vejez) y a otros grupos generacionales, con el fin de informar acerca del envejecimiento normal y sus derivaciones (educación para la vejez). De manera especial la Gerontología Educativa ha focalizado dos áreas específicas: 1) la educación en la vejez, orientada a los mismos mayores; y 2) la educación para la vejez orientada especialmente a personas de otras generaciones, enfoque que actualmente incluye la educación intergeneracional.

En su dimensión teórica la Gerontología Educativa toma como ejes de estudio los cambios intelectuales asociados al proceso de envejecimiento; el desarrollo de adaptaciones instruccionales de acuerdo a las características de los grupos de mayores y a los objetivos de las intervenciones educativas; el estudio de los factores motivacionales ligados a las necesidades de aprendizaje y la participación en actividades educativas (MARTIN GARCIA, 1991).

Un referente importante para el funcionamiento de la CUAM lo constituye su análisis dentro del contexto de la extensión universitaria, constituyendo una manifestación de la relación dialéctica universidad - sociedad que se da en un vínculo cuyo fin es la promoción de la cultura, es una función en la cual deben intervenir todos los factores componentes de la institución de educación superior y como proceso posee su propia metodología: la promoción , 
que constituye un elemento esencial para garantizar que la función extensionista pueda cumplirse y contribuir al cumplimiento de la misión de la universidad en su conjunto; a partir del desarrollo de acciones que se dirigen a la creación de valores culturales, la conservación de los valores creados, la difusión y el disfrute (GONZÁLEZ, G.; GONZÁLEZ, M., 2001). Este proceso se gestiona a partir del Trabajo Sociocultural Universitario, definido como:

[...] el proceso de gestión que desde los presupuestos de la Promoción Sociocultural resuelve en su desarrollo, la contradicción entre la dimensión administrativa y tecnológica de dicho proceso, que permite a los integrantes de la comunidad intra y extrauniversitaria, optimizar y lograr los objetivos propuestos, con un mínimo de recursos, interactuando a partir de su carácter sistémico y de las Leyes de la Pedagogía en el entorno intra y extrauniversitario para brindar la estrategia a seguir en la contribución universitaria al desarrollo sociocultural. (GONZÁLEZ, M., 2002).

\section{Etapas y desarrollo de la CUAM en la provincia Mayabeque}

El surgimiento de la CUAM en la provincia La Habana (actualmente Mayabeque y Artemisa), tuvo como principal objetivo contribuir a desarrollar en el Adulto Mayor la capacidad de influir en su propio desarrollo humano, propiciando la realización de nuevos planes en esta etapa de la vida. Para ello se establecieron un conjunto de funciones entre las que se encuentran (UNAH, 2002):

- Estudio de carácter multidisciplinario del proceso del envejecimiento poblacional y su repercusión.

- Creación de las filiales en los municipios y las aulas que correspondan a su potencial de matrícula según las condiciones territoriales.

- Asesorar a los municipios desde el punto de vista docente metodológico y científico investigativo.

- Elaborar orientaciones y materiales que contribuyan a la calidad del desarrollo del programa de estudio.

- Participar en eventos científicos nacionales e internacionales sobre el tema de envejecimiento.

- Asesorar trabajos de curso en las Sedes Universitarias.

- Coordinación de intercambios intergeneracionales.

- Capacitación sobre temas gerontológicos y de educación de adultos mayores.

- Convocar a los intercambios de experiencias desde la base hasta nivel provincial.

- Tributar al grupo provincial y nacional los resultados del trabajo docente investigativo 
- Atender a los coordinadores municipales así como a los responsables de módulos para garantizar la calidad de la docencia.

La primera etapa de trabajo fue la constitución del grupo gestor, que tendría a su cargo la dirección organizativa de este proyecto social y educacional. El mismo estuvo integrado por representantes de la CTC, APC y MES de la provincia, apoyados en su trabajo por colaboradores de las direcciones de Salud, Trabajo, Cultura, INDER del territorio que tuvieron a su cargo la organización docente de los temas fundamentales que conforman los módulos del programa de estudio.

La estructura del programa desde el punto de vista docente se desarrolla a través de tres sistemas. El primero es el denominado curso básico con duración de un año escolar, dirigido a las personas mayores, y con carácter modular. El segundo sistema docente consiste en los denominados cursos de continuidad, de amplio espectro en su diversidad temática y también dirigida a los adultos mayores, en este caso, a los mayores egresados del curso básico. El tercer sistema es de capacitación y postgrado en el tema gerontológico y materias afines, dirigido a los recursos humanos que participan como profesores en el proceso educativo con mayores.

Teniendo en cuenta el sistema descrito, la segunda etapa se enmarcó en la organización docente del curso básico que cuenta con 42 semanas lectivas, de ellas 36 destinadas a la actividad docente y 2 a la práctica investigativa. Los contenidos del programa se encuentran distribuidos en cinco módulos: Propedéutico, Desarrollo Humano, Educación y Promoción de Salud, Seguridad y Servicio Social, Cultura. Al finalizar este curso los Adultos Mayores deben presentar un trabajo final evaluativo que resuelva un problema práctico o investigue en el desarrollo histórico-cultural de su municipio.

El día primero de octubre, tomando como referente el Día Mundial del Adulto Mayor, se realizó el acto académico central de inauguración del primer curso de la CUAM con la puesta en marcha del curso básico en los 19 territorios de La Habana. De esta forma se oficializaba la apertura de una nueva institución educacional que tendría la responsabilidad de la atención personalizada a la tercera edad desde la labor extensionista de las SUM, cumplimentándose con ello la tercera etapa del proceso.

Durante el desarrollo de los módulos, como parte de la cuarta etapa, se realizaron evaluaciones parciales de la satisfacción de los estudiantes con los contenidos impartidos, identificando como principales indicadores evaluados: el nivel de aceptación y motivación de los temas impartidos, la correspondencia entre las exigencias de esta etapa y la preparación recibida, el accionar del Adulto Mayor a partir de estos cursos en la transformación de su entorno social y familiar. A partir de las fortalezas y debilidades detectadas se estableció un sistema de recomendaciones para continuar trabajando. 
La Cátedra del Adulto Mayor en la provincia de Mayabeque: materialización del trabajo extensionista

La quinta etapa, se identifica con la socialización de los resultados presentados como evaluación final del curso. Para ello se instauró anualmente un evento de carácter provincial denominado en sus inicios GERONTOHABANA, combinando la palabra de la ciencia que atiende al adulto en su educación y el nombre de la provincia. Se acordó convocarlo una vez al año desde el nivel municipal. Los primeros trabajos que se presentaron eran objetos artesanales, tejidos y poemas, pero con el avance del proyecto la situación fue variando; la profundidad de las investigaciones fue en ascenso; a partir de la consolidación del asesoramiento y tutoría por parte de profesores hacia los estudiantes, reflejándose en la participación de varios de estos trabajos en otros eventos nacionales y provinciales obteniendo premios y reconocimientos por su calidad.

Las primeras convocatorias del evento a nivel municipal se efectuaron en Bauta, y en el Instituto Superior Pedagógico Villena. A partir de la tercera edición ha sido su sitio permanente, la Universidad Agraria de La Habana. Por la complejidad de la provincia y la participación masiva al evento, se decidió convocarlo en cuatro regiones de la provincia, con ello se pueden valorar, socializar y discutir un mayor número de trabajos. La distribución por regiones se relaciona a continuación:

- Región 1 (Güines, San José, San Nicolás, Melena y Batabanó)

- Región 2 (Guanajay, Artemisa, Bauta, Caimito y Mariel)

- Región 3 (San Antonio, Güira, Alquízar, Quivicán)

- Región 4 (Jaruco, Santa Cruz, Madruga y Nueva Paz)

Los trabajos comenzaron a responder a temas puntuales abordados durante los módulos del curso básico y a problemas detectados dentro de los territorios. Constituyendo las principales temáticas de interés abordadas:

- Promoción de salud (Proyectos de desarrollo humano, para la prevención del cáncer mamario, del medio ambiente, de las ITS y el VIH; las drogas, el alcoholismo y las enfermedades crónicas como el asma.

- Calidad de Vida (Realización de ejercicios físicos, hábitos alimenticios correctos, asimilación de elementos de otras culturas como el yoga, o el Tay Chi contra el stres).

- Atención Social (Violencia intrafamiliar, niños que no cumplen sus deberes escolares, prostitución, jóvenes desvinculados y exreclusos).

- Identidad (Promoción de la Historia Local, de la obra de los artistas del territorio, artesanía, manualidades y recetas autóctonas).

- Desarrollo cultural (movimiento de aficionados al arte, peñas, tertulias, encuentros intergeneracionales y participación en eventos de diferentes temas) 
La Cátedra del Adulto Mayor en la provincia de Mayabeque: materialización del trabajo extensionista

Llega la división política administrativa y con ella, la definición del ciclo de vida de evento; se consultó con los adultos mayores que estudian en las actuales aulas y por consenso se decidió continuar ahora bajo la denominación de GERONTOVIDA.

Teniendo en cuenta las nuevas transformaciones ocurridas como parte de la nueva distribución política administrativa de la provincia La Habana en Mayabeque y Artemisa, este año 2011 se decide cambiar su nombre por GEREONTOVIDA. Por los resultados mostrados en esta última edición, se decidió convocar a nivel nacional en el próximo año este evento.

La sexta etapa, corresponde a la evaluación final del trabajo de la Cátedra, para ello se diseñaron y aplicaron varios instrumentos de investigación como son encuestas a los Adultos Mayores matriculados en el curso y entrevistas grupales con los profesores y directivos de la CUAM, del mismo modo fueron evaluados los indicadores de permanencia y eficiencia lograda en el curso conjuntamente con la calidad de los trabajos y el impacto social logrado en las comunidades. Todo ello permitió realizar un balance en términos de fortalezas y debilidades que generó la retroalimentación del proceso y la apertura del nuevo ciclo escolar donde aparece en la segunda etapa la organización además del curso de continuantes.

En cuanto al programa de estudio, el cambio más significativo se expresa en la introducción de los denominados ejes temáticos: creatividad, valores, enfoque de género, pensamiento martiano y medio ambiente, a tratar en cada uno de los módulos docentes.

La siguiente figura (Figura 1) muestra el carácter sistémico de cada una de las etapas que fueron descritas con anterioridad.

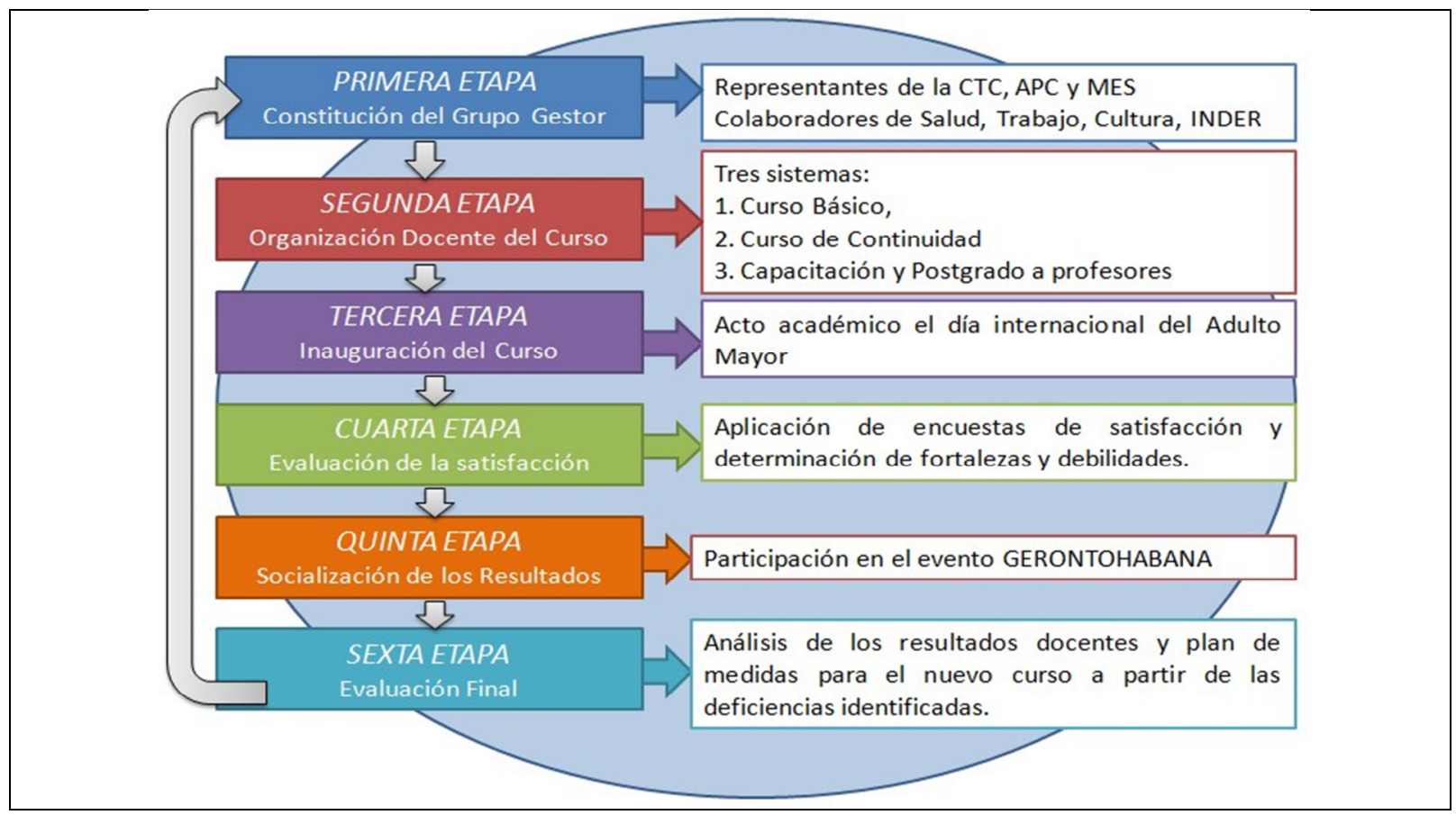

Figura 1. Etapas en el desarrollo de la CUAM en la provincia Mayabeque Fuente: Elaboración propia. 
La Cátedra del Adulto Mayor en la provincia de Mayabeque: materialización del trabajo extensionista

Hoy, a más de una década de iniciada la experiencia del trabajo con el Adulto Mayor desde una Cátedra Universitaria , no se concibe la transformación sociocultural de los territorios sin la impronta de los alumnos de este proyecto; los centros universitarios los sienten suyos y ellos a su vez tienen un marcado sentido de pertenencia por la universidad; poseen su logotipo (premio de un evento del municipio San José, liderado por un graduado de Comunicación Social), su carné acreditativo, sus canciones, sus peñas y por parte de las CUM se observan numerosas acciones de atención a los adultos mayores de las filiales. Profesores adjuntos de las mismas imparten talleres en el curso básico o en el de continuantes, realizan funciones de tutorías a los trabajos de cursos, propician de intercambios intergeneracionales y los envejescentes se convierten en promotores de salud, de cultura e incluso lideran múltiples proyectos para el beneficio social; sobre todo han comenzado a pensar en ellos primero, viviendo intensamente la adultez mayor como una de las etapas del desarrollo humano con potencialidades y debilidades y no el fin de la esperanza.

La tabla (Tabla 1) siguiente resume indicadores importantes que demuestran cómo ha evolucionado el trabajo con el adulto mayor en la provincia.

Tabla 1: Indicadores del desarrollo de la CUAM de Mayabeque en los últimos cinco años.

\begin{tabular}{|c|c|c|c|c|c|c|c|c|c|c|c|}
\hline \multirow{3}{*}{ CURSO } & \multicolumn{6}{|c|}{ MATRÍCULA } & \multicolumn{2}{|c|}{ AULAS } & \multirow{3}{*}{ PROFESORES } & \multirow{3}{*}{$\begin{array}{l}\text { TOTAL DE } \\
\text { GRADUADOS }\end{array}$} & \multirow{3}{*}{$\begin{array}{l}\text { PARTICIPACIÓ } \\
\text { N EN EVENTOS }\end{array}$} \\
\hline & \multicolumn{3}{|c|}{ CURSO BÁSICO } & \multicolumn{3}{|c|}{ CONTINUANTES } & & & & & \\
\hline & $\mathrm{F}$ & M & $\mathrm{T}$ & $\mathrm{F}$ & M & $\mathrm{T}$ & $\mathrm{CB}$ & C & & & \\
\hline $\begin{array}{l}2009- \\
2010\end{array}$ & 209 & 392 & 601 & 321 & 101 & 432 & 20 & 32 & 398 & 526 & 69 \\
\hline $\begin{array}{l}2010- \\
2011\end{array}$ & 204 & 376 & 580 & 519 & 201 & 720 & 22 & 34 & 367 & 429 & 74 \\
\hline $\begin{array}{l}2011- \\
2012\end{array}$ & 394 & 202 & 596 & 840 & 183 & 1023 & 34 & 48 & 401 & 596 & 90 \\
\hline $\begin{array}{l}2012- \\
2013\end{array}$ & 321 & 106 & 427 & 878 & 159 & 1037 & 31 & 40 & 325 & 425 & 147 \\
\hline $\begin{array}{l}2013- \\
2014\end{array}$ & 386 & 137 & 523 & 546 & 233 & 797 & 33 & 43 & 331 & 523 & 146 \\
\hline
\end{tabular}

Fuente: Elaboración propia.

\section{Resultados de la encuesta aplicada para evaluar el funcionamiento de la Cátedra del Adulto Mayor en la provincia Mayabeque}

Para el análisis del funcionamiento de las Cátedras del Adulto Mayor fue aplicada una encuesta a los once coordinadores que atienden las Filiales del Adulto Mayor en la provincia 
Mayabeque, en todos los casos son profesores universitarios. La muestra selecciona fue no probabilística intencional.

El instrumento contó con siete preguntas entre cerradas y abiertas, en algunas de ellas se empleó la escala de Likert para su evaluación. La pregunta 1 estaba enfocada a las posibilidades que ha brindado a los Adultos Mayores la presencia de Filiales Universitarias en los municipios, la siguiente gráfica (Figura 2) muestra los resultados obtenidos. Se puede observar que se reconoce como principales posibilidades la integración social, el conocimiento de esta nueva etapa y el intercambio con otros adultos mayores, siendo las de menor selección la posibilidad de conocer el vínculo universidad/sociedad y la participación en eventos y actividades. Todo ello se justifica a partir de que todavía son insuficientes las actividades que permiten el intercambio abierto de los miembros de las CUAM con la comunidad universitaria, al mismo tiempo los cursos abordan las temáticas desde el aspecto social, biológico y cultural del fenómeno del envejecimiento: sin embargo no se trabaja desde la dimensión extensionista de la universidad y su implicación en el cambio social donde los cursistas pueden jugar un papel más protagónico si se tienen en consideración como promotores socioculturales en cada uno de los territorios.

En la pregunta 2, los encuestados manifiestan, a partir de los 11 ítem empleados, estar de acuerdo en: (100\%) la planificación de los módulos y contenidos de los cursos corresponden a las necesidades del adulto mayor; (100 \%) existe preparación en el claustro de profesores que imparten los módulos; (100\%) existe motivación de los Adultos Mayores en participar en las actividades que promueven las filiales y no estar de acuerdo con: (40\%) existen espacios de intercambio entre los estudiantes de la universidad y los de la filial; (65\%) se ha logrado incorporar a todos los Adultos Mayores con posibilidades del territorio en el programa de la CUAM; el resto fueron evaluados como bastante adecuados. A partir de estos resultados se puede concluir que se necesitan incrementar los espacios para el intercambio intergeneracional y la promoción de este programa en todas las comunidades y barrios apoyándose en las principales organizaciones de masa. 


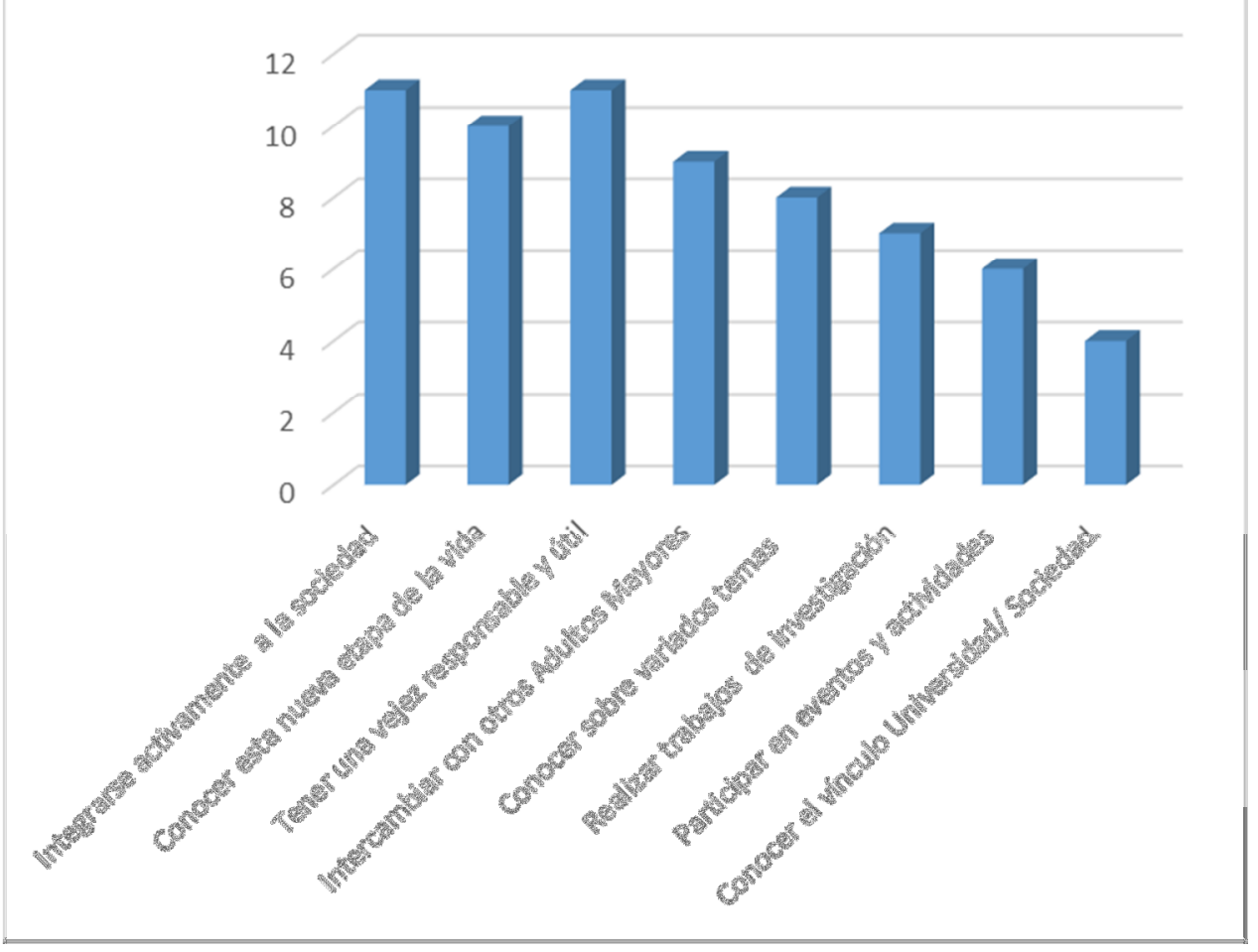

Figura 2. Posibilidades que ha brindado a los Adultos Mayores la presencia de Filiales Universitarias en los municipios.

Fuente: Elaboración propia

En cuanto a la pregunta 3, que se refería a enumerar las principales deficiencias en el funcionamiento de las filiales se encontraron como regularidades: la no existencia de programas que vinculen a adultos mayores con dificultades para acceder a los lugares donde funcionan las aulas, los insuficientes espacios para el intercambio con adultos mayores de otros municipios, en particular se refirieron a que GERONTOVIDA era un evento propicio pero que deberían existir otros de igual tipo que promovieran además de la investigación que se lleva a cabo espacios para el intercambio cultural y grupos de aficionados que presentan algunos territorios.

La pregunta 4 se refería a la importancia que se le confiere a que los profesores tengan dominio de los contenidos de Andragogía, Gerontagogía y Geriatría, de los 9 ítem evaluados los de mayor aceptación fueron: mejor comunicación profesor-adulto mayor; las exigencias en la dirección del aprendizaje se corresponden con las posibilidades cognitivas; las actividades responden a las necesidades y motivos del estudiante y existe mayor participación en clase de los adultos mayores.

En la pregunta 5, referida a las actividades en la que participan, todos se refirieron en primer lugar al evento GERONTOVIDA y el resto son actividades aisladas promovidas por Casas de la Cultura e Instituciones del territorio, siendo las de menos incidencia las organizadas por las propias filiales. Ello evidencia el papel más activo que debe desarrollar las filiales en la organización de actividades dentro de propia organización que tenga en cuenta las particularidades de los miembros y sus intereses. 
En la pregunta 6 cuando se expresan las principales satisfacciones e insatisfacciones que poseen con el funcionamiento de las filiales, se muestran en primer lugar más satisfacciones (12) que insatisfacciones (3), las primeras se pueden resumir en la importancia para que los adultos puedan asumir una vejez responsable y sentirse útil en la sociedad, las segundas a aspectos organizativos puntuales de algunas filiales.

La pregunta 7 y última, se refería a algunas recomendaciones que pudieran servir para el perfeccionamiento de las filiales, dentro de todos los aspectos planteados se seleccionaron por su relevancia los siguientes:

- Utilizar a los adultos mayores en programas de prevención y promoción sociocultural.

- Incrementar la frecuencia en que se realizan los encuentros por ser un momento en que se sienten motivados y alejados de los problemas.

- Incrementar los espacios de comunicación entre la comunidad universitaria y las filiales.

- Incluir algunas temáticas nuevas a los cursos como son la subversión político ideológica, la influencia que desde la tercera edad se puede realizar sobre la juventud en el fortalecimiento de los valores patrios, psicología para ayudar a los hijos en cómo educar a los nietos, etcétera.

\section{Conclusiones}

La CUAM en Mayabeque constituye un programa consolidado con excelentes resultados en la articulación de los tres procesos sustantivos de la universidad: formación, investigación y extensión; sus alumnos han logrado el reconocimiento territorial, provincial y nacional por el impacto de sus investigaciones y la pertinencia de su quehacer para la transformación de la realidad mayabequense.

Las investigaciones en temas de cultura, identidad, historia, patrimonio, salud, medioambiente y prevención social son pertinentes en tanto responden a las problemáticas detectadas en las localidades de la provincia y la socialización de sus resultados se ha producido a través del evento GERONTOHABANA (a partir de marzo 2011, GERONTOVIDA), escenario estratégico para el intercambio entre los Adultos Mayores.

La encuesta aplicada evidencia que el desarrollo de las Filiales del Adulto Mayor en los municipios han tenido un impacto importante en el desarrollo humano de este grupo social, permitiéndoles conocer sobre las particularidades del envejecer para que se asuma como una nueva etapa igualmente útil y feliz. También demuestra la necesidad de perfeccionar el funcionamiento en aras de convertir este espacio en un centro motivador para todos los adultos 
mayores de la localidad, de igual forma establecer métodos y vías para incorporar a los que no pueden asistir a las sedes habituales por diferentes dificultades.

\section{Referências}

CASTRO, F. Discurso pronunciado en el VI Periodo de secciones del Asamblea Nacional del Poder Popular. Granma. La Habana, Cuba, 1984.

FARIÑAS, L. Los retos de envejecer. Granma, 14 nov. 2013, p. 8.

FLECHA, R. Educación de personas adultas: propuestas para los años noventa. Barcelona: El! Roure, 1994.

GARCÍA MARTÍN, A. Ocio activo y bienestar subjetivo en personas mayores. Málaga: Universidad de Málaga: Servicio de Publicaciones e Intercambio Científico, 1995.

GLENDENNING, F. Teaching and learning in later life: theoretical implications. Aldershot: Arena, 1990.

GONZÁLEZ, G. Un Modelo de extensión universitaria: su aplicación a la cultura física y el deporte. Tesis. Doctorado. Instituto Superior de Cultura Física Manuel Fajardo, La Habana, Cuba, 1996.

GONZÁLEZ, G.; GONZÁLEZ, M. Programa Nacional de Extensión Universitaria. La Habana, Cuba, 2001.

JARVIS, P. The sociology of adult and continuing education. Londres: Croom Helm, 1985.

KNOWLES, M. La Práctica Moderna de la Educación de Adultos. Chicago, USA: Paidós, 1980.

KNOWLES, M. Andragogía no Pedagogía. Centro Regional de Educación de Adultos. Temas de Educación de Adultos, año I, n. 2, Caracas, Venezuela, 1983.

ORGANIZACIÓN MUNDIAL DE LA SALUD. El movimiento mundial en pro del envejecimiento activo. Ginebra, Suiza, 1999.

OROSA, T. La Tercera Edad y la familia: una mirada desde el adulto mayor. La Habana, Cuba: Editorial Félix Varela, 2001.

PETERSON, D. Educational Gerontology: The state of the art. Educational Gerontology, n. 1, 1976.

TURNER, L. Documento del Seminario impartido en la I Reunión Nacional de Cátedras del Adulto Mayor. Impreso. La Habana, Cuba, 2001.

UNESCO. Documento para el Cambio y Desarrollo de la Educación Superior. Paris: UNESCO, 1995.

UNIVERSIDAD AGRARIA DE LA HABANA. Lineamientos para el trabajo con las CUAM de la provincia La Habana. Impreso. La Habana, Cuba, 2002. 
The Elderly Groups in the Mayabeque Province: the materialization of an extension work

\section{ABSTRACT}

As part of an extensive work carried out by the University in the community, The Elderly Groups were founded, where people of the age prepare for a responsible and complete oldness. The objective of this paper is to present the experience of the UNAH with the functioning of these elderly groups in the province of Mayabeque. The experiences presented in the previous editions of GERONTOHABANA by the members of these groups have been taken as references for the socio-cultural work developed by the university with the involvement of the elderly as promoters of the extensive process in the community.

\section{Keywords}

Elderly Groups; Elderly - Gerontology; University Socio-Cultural Work.

Original submetido em: 26 out. 2015

Aceito para publicação em: 8 dez. 2015
A Cátedra do Idoso na província de Mayabeque: materialização do trabalho extensionista

\section{RESUMO}

Como parte do trabalho extensionista da universidade para a comunidade surgem as Cátedras del Adulto Mayor (CUAM). Nelas as pessoas da terceira idade se preparam para uma velhice responsável e plena. 0 presente artigo tem como objetivo apresentar a expêriencia da Universidad Agraria de La Habana (UNAH), no funcionamento da referida Cátedra no estado de Mayabeque. Foram tomados como referentes os resultados da enquete aplicada aos coordenadores e os trabalhos apresentados por seus membros nas edições do evento GERONTOHABANA. Evidencia o trabalho sociocultural universitário desenvolvido no território com a participação de idosos como promotores do processo de extensão.

\section{Palavras-chave}

Cátedra do Idoso; Gerontologia educativa; Trabalho Sociocultural Universitário.

Sobre os autores:

\section{Milagros de la C. Socas Reinoso}

Asesora de la Rectora, Universidad Agraria de La Habana "Fructuoso Rodríguez Pérez".

\section{Alexis Torres Alonso}

Investigador del Centro de Estudios de la Educación Superior Agropecuaria, Universidad Agraria de La Habana "Fructuoso Rodríguez Pérez". 